Mathematical Research Letters 5, 149-163 (1998)

\title{
NORMAL FORMS AND INVARIANT GEOMETRIC STRUCTURES FOR DYNAMICAL SYSTEMS WITH INVARIANT CONTRACTING FOLIATIONS
}

\author{
M. Guysinsky and A. Katok
}

\begin{abstract}
We present a certain version of the "non-stationary" normal forms theory for extensions of topological dynamical systems (homeomorphisms of compact metrizable spaces) by smooth $\left(C^{\infty}\right)$ contractions of $\mathbb{R}^{n}$. The central concept is a notion of a sub-resonance relation which is an appropriate generalization of the notion of resonance between the eigenvalues of a matrix which plays a similar role in the local normal forms theory going back to Poincaré and developed in the modern form for $C^{\infty}$ maps by S. Sternberg and K.-T. Chen. Applicability of these concepts depends on the narrow band condition, a certain collection of inequalities between the relative rates of contraction in the fibers. One of the ways to formulate our first conclusion (the sub-resonance normal form theorem) is to say that there is a continuous invariant family of geometric structures in the fibers whose automorphism groups are certain finite-dimensional Lie groups. Our central result is the joint normal form for the centralizer for an extension satisfying the narrow band condition. While our non-stationary normal forms are rather close to the normal forms in a neighborhood of an invariant manifold, studied in the literature, the centralizer theorem seems to be new even in the classical local case. The principal situation where our analysis applies is a smooth system on a compact manifold with an invariant contracting foliation. In this case we also establish smoothness of the sub-resonance normal form along the fibers. The principal applications so far are to local differentiable rigidity of algebraic Anosov actions of higher-rank abelian groups and algebraic Anosov and partially hyperbolic actions of lattices in higher-rank semi-simple Lie groups.
\end{abstract}

\section{Extensions of continuous maps by contractions}

1.1. Linear extensions. Let $X$ be a compact metric space, $f: X \rightarrow X$ a homeomorphism (continuous dynamical system), $V$ a vector bundle over $X$ with projection $\pi: V \rightarrow X, F: V \rightarrow V$ a continuous invertible linear extension of $f$. Let us fix a continuous family of Riemannian metrics in the fibers. Extension $F$ is called a contraction if $\|D F\|<1$. Consider the induced operator $F^{*}$ in the Banach space of continuous sections of $V$ provided with the uniform norm for

$$
F^{*} v(x)=F\left(v\left(f^{-1} x\right) .\right.
$$

Received October 5, 1997.

The first author is supported by the Pritchard Dissertation Fellowship at the Pennsylvania State University.

The second author is partially supported by NSF grants DMS-9404061 and DMS-9704776. 
Under a very mild assumption that non-periodic points of $f$ are dense the spectrum of the operator $F^{*}$ is the union of finitely many closed annuli centered at the origin. Hence the characteristic set of $F$,

$$
\chi(F)=\left\{\lambda \in \mathbb{R}_{-}: \exp \lambda \in \operatorname{sp} F^{*}\right\}
$$

is the union of finitely many intervals. Let us denote these intervals by $\Delta_{1}, \ldots \Delta_{l}$. Let $\Delta_{i}=\left[\lambda_{i}, \mu_{i}\right]$ and assume that the intervals are ordered in increasing order so that $\lambda_{i+1}>\mu_{i}$. Let us assume that the space $X$ is connected or that the map $f$ is topologically transitive. Then the bundle $V$ splits into the direct sum of $F$-invariant subbundles $V_{1}, \ldots, V_{l}$ such that $\chi(F) \uparrow V_{i}=\Delta_{i}, \quad i=1, \ldots, l[\mathrm{~K} 1]$. Let $m_{i}$ be the dimension of the sub-bundle $V_{i}$.

Denote the given scalar product in the fiber $V_{x}$ over the point $x$ by $\langle\cdot, \cdot\rangle_{x}$. Given $\epsilon>0$ we will call the standard $\epsilon$-Lyapunov scalar product (or metric) the scalar product $\langle\cdot, \cdot\rangle_{x, \epsilon}^{\prime}$ in $V_{x}$ defined as follows (Cf. [K2,Section 1.1]): For $u, v \in V_{i}(x), i=1, \ldots, l$ :

$$
\begin{aligned}
<u, v & >_{x, \epsilon}^{\prime}=\sum_{n=0}^{\infty}<F^{-n} u, F^{-n} v>_{F^{-n} x} \exp 2\left(\lambda_{i}-\epsilon\right) n \\
& +\sum_{n=1}^{\infty}<F^{n} u, F^{n} v>_{F^{n} x} \exp -2\left(\mu_{i}+\epsilon\right) n .
\end{aligned}
$$

Our assumption about the spectrum implies convergence of this series. For $u \in V_{i}(x), v \in V_{j}(x), i \neq j$ we set $\langle u, v\rangle_{x, \epsilon}^{\prime}=0$. It immediately follows from (1.1) that for $u \in V_{x}$,

$$
\exp \left(\lambda_{i}-\epsilon\right)\|u\|_{x, \epsilon}^{\prime} \leq\|F u\|_{f x, \epsilon}^{\prime} \leq \exp \left(\mu_{i}+\epsilon\right)\|u\|_{x, \epsilon}^{\prime} .
$$

In particular, if the spectral radius $\exp \mu_{l}$ is less than 1 and $\epsilon$ is chosen small enough, then $F$ is a contraction with respect to the standard $\epsilon$-Lyapunov metric.

Definition 1.1. Extension $F$ has a narrow band spectrum if

$$
\mu_{i}+\mu_{l}<\lambda_{i}
$$

for $i=1, \ldots, l$.

From now on we assume that this condition is satisfied. Represent $\mathbb{R}^{m}$ as the direct sum of subspaces $V_{1}, \ldots, V_{l}$ of dimension $m_{1}, \ldots, m_{l}$ correspondingly and let $\left(t_{1}, \ldots, t_{l}\right)$ be the corresponding coordinate representation of a vector $t \in \mathbb{R}^{m}$. Let $P: \mathbb{R}^{m} \rightarrow \mathbb{R}^{m} ;\left(t_{1}, \ldots, t_{l}\right) \mapsto\left(P_{1}\left(t_{1}, \ldots, t_{l}\right), \ldots, P_{l}\left(t_{1}, \ldots, t_{l}\right)\right)$ be a polynomial map preserving the origin. We will say that the map $P$ is of sub-resonance type if it has non-zero homogeneous terms in $P_{i}\left(t_{1}, \ldots, t_{l}\right)$ with degree of homogeneity $s_{j}$ in the coordinates of $t_{j}, i=1, \ldots, l$ only if

$$
\lambda_{i} \leq \sum_{j \neq i} s_{j} \mu_{j} .
$$


Let as notice that the notions of a homogeneous polynomial and degree of homogeneity in a Euclidean space are invariant under the action of the linear changes of coordinates. Thus the notion of a map of sub-resonance type depends only on the decomposition $\mathbb{R}^{m}=\bigoplus_{i=1}^{l} V_{i}$, but not on a choice of coordinates in each component of this decomposition. We will assume that this decomposition is fixed and will omit dependence on the decomposition in our notation.

We will call any inequality of type (1.3) a sub-resonance relation. There are always sub-resonance relations of the form $\lambda_{i} \leq \mu_{j}$ for $j=i, \ldots, l$. They correspond to the linear terms of the polynomial. We will call such relations trivial. The narrow band condition (1.2) guarantees that for any non-trivial sub-resonance relation $s_{j}=0$ for $j=1, \ldots, i-1$.

Thus there are only finitely many different sub-resonance relations and hence polynomial maps of sub-resonance type form a finite-dimensional vector space. It is easy to see that any polynomial map of the sub-resonance type with invertible derivative at the origin is invertible. Let us denote by $P_{\lambda, \mu}$ the set of polynomial maps of the sub-resonance type with invertible derivative at the origin and by $G_{\lambda, \mu}$ the group generated by $P_{\lambda, \mu}$.

We will call the elements of the group $G_{\lambda, \mu}$ sub-resonance generated polynomial maps. In certain cases $P_{\lambda, \mu}$ is already a group, i.e. $P_{\lambda, \mu}=G_{\lambda, \mu}$. In particular, if the whole spectrum is sufficiently narrow i.e if $\lambda_{1}>2 \mu_{l}$, then there are no non-trivial sub-resonance relations and hence $P_{\lambda, \mu}=G_{\lambda, \mu}=G L(m, \mathbb{R})$, the group of linear automorphisms of $\mathbb{R}^{m}$. The next simplest case appears in the perturbation of the point spectrum with $2: 1$ resonance. In this case $l=2$ and $\lambda_{1}>\max \left\{3 \mu_{2}, \mu_{1}+\mu_{2}\right\}$. In this case the only possible non-trivial sub-resonance relation is $\lambda_{1} \leq 2 \mu_{2}$. The group $G_{\lambda, \mu}=P_{\lambda, \mu}$ consists of quadratic maps of the form

$$
P\left(t_{1}, t_{2}\right)=\left(L_{1} t_{1}+Q\left(t_{2}, t_{2}\right), L_{2} t_{2}\right),
$$

where $L_{1}$ and $L_{2}$ are linear maps and $Q$ is a quadratic form. The above two cases are the only ones which appear in the consideration of small perturbations of Weyl chamber flows (cf $[\mathrm{KS}]$ ). Yet another case is that of the point spectrum i.e. when $\lambda_{i}=\mu_{i} i=1, \ldots, l$.

Lemma 1.1. $P_{\lambda, \lambda}$ is a group.

Proof. First we show that $P_{\lambda, \lambda}$ is closed under the operation of superposition.

If the polynomial maps $F, G$ belong to $P_{\lambda, \lambda}$ and $F=\left(F_{1}, \ldots, F_{l}\right), G=$ $\left(G_{1}, \ldots, G_{l}\right)$, then

$$
F \circ G=\left(F_{1} \circ G, F_{2} \circ G, \ldots, F_{l} \circ G\right),
$$

and we have to prove that $F_{i} \circ G$ is of sub-resonance type. The polynomial map $F_{i}$ includes only such terms $x_{1}^{s_{1}} \ldots x_{i}^{s_{i}}$ that

$$
\lambda_{i} \leq \sum_{j} s_{j} \lambda_{j}
$$


If we take superposition of a term like this and $G$ we obtain a sum that consists of terms $y_{1}^{s_{1}} y_{2}^{s_{2}} \ldots y_{i}^{s_{i}}$ where $y_{j}=x_{1}^{s_{1}^{j}} x_{2}^{s_{2}^{j}} \ldots x_{i}^{s_{i}^{j}}$ is a sub-resonance monomial for $j$-th coordinate and therefore satisfies the following condition:

$$
\lambda_{j} \leq \sum_{k} s_{k}^{j} \lambda_{k}
$$

The only thing we have to check is if

$$
y_{1}^{s_{1}} y_{2}^{s_{2}} \ldots y_{i}^{s_{i}}=\left(x_{1}^{s_{1}^{1}} \ldots x_{i}^{s_{i}^{1}}\right)^{s_{1}}\left(x_{1}^{s_{1}^{2}} \ldots x_{i}^{s_{i}^{2}}\right)^{s_{2}}\left(x_{1}^{s_{1}^{i}} \ldots x_{i}^{s_{i}^{i}}\right)^{s_{i}}
$$

satisfies the sub-resonance condition:

$$
\lambda_{i} \leq \sum_{k} \sum_{j} s_{k}^{j} s_{j} \lambda_{k}
$$

Indeed, using first (1.5) and then (1.4) we have

$$
\sum_{k} \sum_{j} s_{k}^{j} s_{j} \lambda_{k}=\sum_{j} \sum_{k} s_{j} s_{k}^{j} \lambda_{k} \geq \sum_{j} s_{j} \lambda_{j} \geq \lambda_{i} .
$$

To prove that $P_{\lambda, \lambda}$ is a group, we also have to prove that if $F \in P_{\lambda, \lambda}$, then $F^{-1} \in P_{\lambda, \lambda}$.. We will show that $F^{-1}$ can be found as the product of maps that belong to $P_{\lambda, \lambda}$ and therefore it belongs to $P_{\lambda, \lambda}$ itself.

First multiply $F$ from the right by the inverse of its linear part. We will obtain a map $F_{1}$ that can be represented as $I d+S_{2}+S_{>2}$, where the polynomial map $S_{2}$ is a part of $F_{1}$ that contains all terms of degree 2 and $S_{>2}$ all the other terms ( degree greater than 2). We can see that the map $P_{2}=\left(I d-S_{2}\right) \in P_{\lambda, \lambda}$ and the map $F_{2}=F_{1} \circ P_{2}=I d+S_{2}-S_{2}+S_{>2}=I d+S_{3}+S_{>3}$ also belong to $P_{\lambda, \lambda}$. In the same way we can eliminate all the terms of degree 3 and so on. At each step we get a polynomial map that belongs to $P_{\lambda, \lambda}$. Since the degree of any polynomial map from $P_{\lambda, \lambda}$ is bounded by $\frac{\lambda_{l}}{\lambda_{1}}$, after a finite number of steps we will obtain $F_{n}=I d$. That implies $F \circ P_{1} \circ P_{2} \ldots \circ P_{n}=I d$ and therefore $F^{-1}=P_{1} \circ P_{2} \ldots \circ P_{n}$. Each of $P_{i} \in P_{\lambda, \lambda}$ and hence their product belongs to $P_{\lambda, \lambda}$.

On the other hand, here is a simple example showing that $P_{\lambda, \mu}$ is not necessarily a group.

Example. Let $l=3, \lambda_{1}=\mu_{1}=-3.9, \lambda_{2}=-2.1, \mu_{2}=-1.9, \lambda_{3}=$ $-1.1, \mu_{3}=-1$.

We can see that the only non-trivial sub-resonance relations are $\lambda_{2} \leq 2 \mu_{3}$ and $\lambda_{1} \leq 2 \mu_{2}$. We also notice that $\lambda_{1} \leq 4 \mu_{3}$ is not a sub-resonance relation. However, if we consider a composition of two maps $\left(x_{1}, x_{2}+x_{3}^{2}, x_{3}\right)$ and $\left(x_{1}+x_{2}^{2}, x_{2}, x_{3}\right)$ of sub-resonance type we will obtain a map $\left(x_{1}+x_{2}^{2}+2 x_{2} x_{3}^{2}+x_{3}^{4}, x_{2}+x_{3}^{2}, x_{3}\right)$. This map has a term $x_{3}^{4}$, that corresponds to the sub-resonance $\lambda_{1} \leq 4 \mu_{3}$.

If we want to have a group, we have to add to the relations that define the vector space $P_{\lambda, \mu}$ additional relations obtained by composition of relations we have already had. Only a finite number of relations have to be added. 
Proposition 1.1. The group $G_{\lambda, \mu}$ is finite-dimensional. More specifically, there exists a number $N=N(\lambda, \mu)$ such that all elements on the group $G_{\lambda, \mu}$ are polynomial maps of degree at most $N$.

Proof. Any group $P_{\lambda, \lambda}$ is finite dimensional. To prove that $G_{\lambda, \mu}$ is finite dimensional, we construct a vector $\lambda^{\prime}$ such that $P_{\lambda, \mu}$ is a subgroup of $P_{\lambda^{\prime}, \lambda^{\prime}}$ and so $G_{\lambda, \mu}$ is also a subgroup of $P_{\lambda^{\prime}, \lambda^{\prime}}$.

We define $\lambda^{\prime}$ as $\lambda_{l}^{\prime}=\lambda_{l}, \lambda_{i}^{\prime}=\lambda_{i} \frac{\lambda_{l}}{\mu_{l}} \frac{\lambda_{l-1}}{\mu_{l-1}} \ldots \frac{\lambda_{i+1}}{\mu_{i+1}}$. It is easy to see that if

$$
\lambda_{i} \leq \sum_{i \neq j} s_{j} \mu_{j}=\sum_{i<j} s_{j} \mu_{j}
$$

then

$$
\lambda_{i} \frac{\lambda_{l}}{\mu_{l}} \frac{\lambda_{l-1}}{\mu_{l-1}} \ldots \frac{\lambda_{i+1}}{\mu_{i+1}} \leq \sum_{i<j} s_{j} \mu_{j} \frac{\lambda_{l}}{\mu_{l}} \ldots \frac{\lambda_{j}}{\mu_{j}}=\sum_{i<j} s_{j} \lambda_{j} \frac{\lambda_{l}}{\mu_{l}} \ldots \frac{\lambda_{j+1}}{\mu_{j+1}}
$$

or,

$$
\lambda_{i}^{\prime} \leq \sum_{i<j} s_{j} \lambda_{j}^{\prime}
$$

This means that $P_{\lambda, \mu} \subset P_{\lambda^{\prime}, \lambda^{\prime}}$. Then $G_{\lambda, \mu}$ is a subgroup of $P_{\lambda^{\prime}, \lambda^{\prime}}$ and all the elements in the group $G_{\lambda, \mu}$ have degree at most

$$
\frac{\lambda_{1}^{\prime}}{\lambda_{l}^{\prime}}=\frac{\lambda_{1}}{\lambda_{l}} \frac{\lambda_{l}}{\mu_{l}} \frac{\lambda_{l-1}}{\mu_{l-1}} \ldots \frac{\lambda_{2}}{\mu_{l-2}}=\frac{\lambda_{1}}{\mu_{l}} \frac{\lambda_{l-1}}{\mu_{l-1}} \ldots \frac{\lambda_{2}}{\mu_{2}} .
$$

We can also construct the group $P_{\lambda, \mu}$ explicitly by adding new "allowed relations". We will sketch this construction and leave details to the reader. We call a relation for $i$-th coordinate any $l$-tuple $\left(s_{1}, \ldots s_{l}\right)$ with non-negative integer entries with at least one non-zero entry . We will say that a relation $\left(u_{1}, \ldots u_{l}\right)$ for $i$-th coordinate is less or equal to a relation $\left(s_{1}, \ldots, s_{l}\right)$ for $i$-th coordinate if and only if $u_{1} \geq s_{1},\left(u_{1}+u_{2}\right) \geq\left(s_{1}+s_{2}\right), \ldots,\left(u_{1}+\ldots+u_{l}\right) \geq\left(s_{1}+\ldots s_{l}\right)$. We call a composition of two relations $\left(s_{1}, \ldots s_{l}\right)$ for $i$-th coordinate and $\left(u_{1}, \ldots u_{l}\right)$ for $j$-th coordinate the relation for $i$-th coordinate we get from the compositions of two monomials $x_{1}^{s_{1}} \ldots x_{l}^{s_{l}}$ and $x_{j}=x_{1}^{u_{1}} \ldots x_{l}^{u_{l}}$. So their composition will be a relation $\left(s_{1}+s_{j} u_{1}, \ldots, s_{j} u_{j}, \ldots, s_{l}+s_{j} u_{l}\right)$.

We will see that for any group $P_{\lambda, \mu}$ one can define a list of allowed relations for each coordinate such that an invertible polynomial map $\left(P_{1}, \ldots, P_{i}\right)$ belongs to $P_{\lambda, \mu}$ only if for any non-zero homogeneous term in $P_{i}\left(t_{1}, \ldots, t_{i}\right)$ with the degree of homogeneity $s_{j}$ in the coordinate $t_{j}$ the relation $\left(s_{1}, \ldots, s_{l}\right)$ is allowed for $i$-th coordinate.

We call a list of allowed relations complete if any relation for $i$-th coordinate that is less or equal to allowed one for $i$-th coordinate is also allowed. We call a list of allowed relations closed if the corresponding set of allowed polynomial maps is closed under the operation of composition. Then this set is a group 
because we can repeat the proof of Lemma 1.1. If a list of relations is complete, then it is closed if and only if the composition of maximal relations (maximal with respect to the defined partial order) belong to the list. We call a list of allowed relations upper-triangular if any allowed relation for $i$-th coordinate has $s_{1}=s_{2}=\ldots=s_{i}=0$ or $s_{1}=s_{2}=\ldots=s_{i-1}=0 s_{i}=1$ and $s_{i+1}=\ldots=s_{l}$. The composition of two upper-triangular relations $r_{1}$ for $i$-th coordinate and $r_{2}$ for $j$-th coordinate, where $i<j$, will have the same first $j$ coordinates as $r_{1}$. A set of relations of the sub-resonance type is complete, triangular but may be not closed. To make it closed the following procedure is suggested. We don't add any new relations for coordinates $l$ and $(l-1)$. For coordinate $(l-2)$ we find all maximal relations and take their compositions with maximal relations for coordinate $(l-1)$. If we get a new relation we add this relation and all lesser relations to the list for the coordinate $(l-2)$. Because of the triangular structure all changes will happen at $l$-th coordinate. Then we repeat these operations considering coordinate $l$ instead of $(l-1)$. It is easy to prove then that

(1) The new list is complete.

(2) The new list is closed if we consider compositions of relations for coordinates $l-2, l-1, l$.

(3) The new list generates a set of polynomial maps that is a subset of $P_{\lambda, \mu}$.

(4) The new list is upper-triangular.

If we repeat it for coordinate $(l-3)$, then for $(l-4), \ldots 1$. we will get a list of relations that is complete, closed and generates a group that is a subgroup of $P_{\lambda, \mu}$ so must coincide with $P_{\lambda, \mu}$.

1.2. Non-linear germ extensions. In the same setup as in the previous subsection let $U$ be a neighborhood of the zero section in $V$. We consider an extension map $\mathcal{F}: U \rightarrow V$ which is continuous, smooth (usually $C^{\infty}$ ) along the fibers and preserves the zero section. We will denote the derivative of $\mathcal{F}$ in the fiber direction at the zero section by $D \mathcal{F}_{0}$. It is a linear extension of $f$. As before, fix a continuous family of Riemannian metrics in the fibers. Extension $\mathcal{F}$ is called a contraction if $D \mathcal{F}_{0}$ is a contraction.

The following simple remark can be useful sometimes. If a map $F: V \rightarrow V$ preserves fibers and is a contraction along fibers with respect to a fixed Riemannian metric, then there exists a unique invariant section and thus we are in the situation described above. This follows from the fact that our map induces a contracting map in the space of all continuos sections.

We will consider the following natural notion of conjugacy for extensions of this kind. Two extensions are conjugate if there exists a continuous family of local $C^{\infty}$ diffeomorphisms of the fibers $V(x)$, preserving the origin which transforms one extension into the other. The following "normal form" theorem asserts that any extension by contractions whose derivative at the zero section has the narrow band spectrum is conjugated to an extension which acts in the fibers by polynomials of sub-resonance type. 
Theorem 1.2 (sub-resonance normal form). Suppose the extension $\mathcal{F}$ is a contraction and the linear extension $D \mathcal{F}_{0}$ has a narrow band spectrum determined by the vectors $\lambda=\left(\lambda_{1}, \ldots, \lambda_{l}\right)$ and $\mu=\left(\mu_{1}, \ldots, \mu_{l}\right)$.

There exists an extension $\tilde{\mathcal{F}}$ conjugated to $\mathcal{F}$ such that for every $x \in X$,

$$
\tilde{\mathcal{F}}\left\lceil V(x): \bigoplus_{i=1}^{l} V_{i}(x) \rightarrow \bigoplus_{i=1}^{l} V_{i}(f(x))\right.
$$

is given by a polynomial map of sub-resonance type.

The proof of this theorem follows one of the usual schemes in the normal form theory. In particular, it is parallel to the proof of the non-stationary normal form theorem [K2, Theorem 2.1] for extensions of measure-preserving transformations. It includes three steps:

Step 1 Finding a formal coordinate change, i.e. the Taylor series at the zero section for the desired coordinate change. Naturally, the coefficients should depend continuously on the base point. This is the most essential step which is discussed in detail below.

Step 2 Constructing a continuous family of smooth $\left(C^{\infty}\right)$ coordinate changes in $V(x), x \in X$ for which the formal power series found at Step 1 is the Taylor series at the zero section. This follows directly from a parametric version of [KH, Proposition 6.6.3]. For a detailed argument see [G].

Step 3 The coordinate change constructed at Step 2 conjugates our extension with an extension which is $C^{\infty}$ tangent to the derivative extension. We show that any two $C^{\infty}$ tangent contracting extensions are conjugate via a $C^{\infty}$ coordinate change $C^{\infty}$ tangent to identity. This is achieved via a parametric version of the "homotopy trick". See [KH,Theorem 6.6.5], where this method is carried out in detail in the local case. In $[\mathrm{G}]$ one can find a detailed proof for that step applicable also to the case of sufficiently high finite differentiability, but based on a somewhat different approach.

Step 1. Let $D \mathcal{F}_{0} \uparrow V_{i}(x):=\Phi_{i}(x)$. We will look inductively for successive jets of the desired coordinate change at the zero section to eliminate all but sub-resonance terms of higher and higher order. Since $D \mathcal{F}_{0}$ has the block form, nothing needs to be done for linear terms. At the $n$th inductive step we start from the extension whose $n$th jets are polynomials of sub-resonance type. We will find a coordinate change with the identity linear part and polynomial homogeneous non-linear part of degree $n+1$. Composing this coordinate change with the result from the previous steps would not change the lower order terms. The $t_{i}$ component $C_{x}\left(t_{1}, \ldots, t_{l}\right)$ of the coordinate change with degree of homogeneity $s_{j}$ in the coordinates of $t_{j}, j=1 \ldots, l$ satisfies the following functional equation

$$
\Phi_{i}(x) C_{x}\left(t_{1}, \ldots, t_{l}\right)-C_{f(x)}\left(\Phi_{1}(x) t_{1}, \ldots, \Phi_{l}(x) t_{l}\right)=H_{x}(t),
$$

where $H_{x}(t)$ is a known function determined by the previous steps of the process. This equation is the non-stationary counterpart for the equation for the 
non-resonance coefficients in the Taylor series of the formal coordiante change eliminating non-resonance terms in the local problem (See, for example, $[\mathrm{KH}$, (6.6.1)] and comments thereof). The main difference is that while in the local situation the equation is just algebraic, in our case it is functional. To derive it one writes the coordiante change as the identity plus the sum of homogeneous parts with coefficients depending on $x$ and calculates the corresponding homogeneous terms at the point $f(x)$. Since the only modification of the part of degree $n$ comes from composing the linear part of the map with the corresponding homogeneous term in the coordinate change we deduce that the change is equal exactly to left-hand part of the equation. The right-hand part contains the term which appeared as the result of previous changes of coordinates.

Let for $t=\left(t_{1}, \ldots, t_{l}\right), \quad \Phi(t)=\left(\Phi_{1}\left(t_{1}\right), \ldots, \Phi_{l}\left(t_{l}\right)\right)$. In the non sub-resonance case we can solve this equation by the "telescoping sum"

$$
C_{x}(t)=\sum_{n=0}^{\infty} \Phi_{i}^{-1}(x) \ldots \Phi_{i}^{-1}\left(f^{n}(x)\right) H_{f^{n}(x)}\left(\Phi\left(f^{n-1}(x)\right) \ldots \Phi(f(x))(\Phi(x))(t)\right),
$$

where the series in the right-hand part converges exponentially due to inequality (1.3). In the sub-resonance case we do nothing. The sub-resonance terms of degree $n+1$ do not change at this and successive steps. Notice however that they were likely to have changed at the previous steps. Thus, while the type of normal form is determined by the first jet (the derivative extension), coefficients of its $n$th jet depend on the $(n-1)$ st jet of the original extension.

While it seems very probable that our version of the concept of non-stationary normal form never appeared in the literature before and even its key ingredient, the notion of sub-resonance relation, is probably original, our approach is somewhat close to the study of normal forms near an invariant manifold for a smooth dynamical system. There is a considerable number of publications on the subject; in particular [BK] has some results as well as references. We should mention in this context the 1991 Ph.D thesis (written under the supervision of the second author) and two papers by D. DeLatte ([DL1, DL2]) where nonstationary normal forms for extensions with hyperbolic area-preserving maps in dimension two in the fibers are studied. This is a very special case: there are infinitely many resonances, but in the normal form there is also a constant of motion. So while the normal forms form an infinite-dimensional group, it splits into hyperbolic translations on each invariant hyperbola.

On the other hand, our next theorem which asserts that the sub-resonance normal form is inherited by any smooth extension ( not necessarily by contractions) seems to be new, even in the classical local case, i.e. when $X$ is a single point and $f$ is the identity map. At least we were neither able to find any published result of that kind ( admittedly, the literature on the subject of local normal forms is vast and we perused only a small potion of it); nor, more importantly, any of the several experts in the normal form theory whom we consulted 
mentioned any "folklore" result of that kind known to specialists.

Theorem 1.3 (Centralizer for sub-resonance maps). Suppose $g$ is a homeomorphism of the space $X$ commuting with $f$ and $\tilde{\mathcal{G}}$ is an extension of $g$ by $C^{\infty}$ diffeomorphisms of the fibers (not necessarily contractions) commuting with an extension $\tilde{\mathcal{F}}$ satisfying the assertion of Theorem 1.2. Then $\tilde{\mathcal{G}}$ :

$$
\tilde{\mathcal{G}} \uparrow V(x): \bigoplus_{i=1}^{l} V_{i}(x) \rightarrow \bigoplus_{i=1}^{l} V_{i}(g(x))
$$

is sub-resonance generated polynomial map.

Proof. The proof of this theorem consists of two steps:

1. Proving that the Taylor series of $\tilde{\mathcal{G}}$ at 0 are sub-resonance generated polynomial maps.

2. Proving that the map $\tilde{\mathcal{G}}$ coincides with its Taylor series.

Step 1. Let us denote the Taylor series of $\tilde{\mathcal{F}}$ and $\tilde{\mathcal{G}}$ at the origin by $F(x)$ and $G(x)$ and the homogeneous parts of $F(x)$ and $G(x)$ of degree $k$ by $F_{k}(x)$ and $G_{k}(x)$ correspondingly. Let $J_{k}()$ be the k-th jet. We know that the maps $\tilde{\mathcal{F}}$ and $\tilde{\mathcal{G}}$ commute so

$$
G\left(f^{n}(x)\right) \circ F^{n}(x)=F^{n}(g(x)) \circ G(x) .
$$

It implies that for any $k$ :

$$
J_{k}\left(G\left(f^{n}(x)\right) \circ F^{n}(x)\right)=J_{k}\left(F^{n}(g(x)) \circ G(x)\right) .
$$

In particular for $k=1$ we have:

$$
G_{1}\left(f^{n}(x)\right) \circ F_{1}^{n}(x)=F_{1}^{n}(g(x)) \circ G_{1}(x) .
$$

We know that for any $x$ the linear map $F_{1}(x)$ has a block form so we denote

$$
\begin{gathered}
F_{1}^{n}(x)=F_{1}\left(f^{n-1}(x)\right) \circ \ldots \circ F_{1}(x)=A=\left(\begin{array}{cccc}
A_{1} & 0 & \ldots & 0 \\
0 & A_{2} & \ldots & 0 \\
\vdots & \vdots & \ddots & \vdots \\
0 & 0 & \ldots & A_{l}
\end{array}\right) \\
F_{1}^{n}(g(x))=F_{1}\left(f^{n-1}(g(x))\right) \circ \ldots \circ F_{1}(g(x))=B=\left(\begin{array}{cccc}
B_{1} & 0 & \ldots & 0 \\
0 & B_{2} & \ldots & 0 \\
\vdots & \vdots & \ddots & \vdots \\
0 & 0 & \ldots & B_{l}
\end{array}\right)
\end{gathered}
$$


where due to our assumption on the spectrum

$$
\exp \left(\lambda_{i}-\epsilon\right)\left\|t_{i}\right\|_{x, \epsilon}^{\prime} \leq\left\|A_{i}\left(t_{i}\right)\right\|_{f^{n}(x), \epsilon}^{\prime} \leq \exp \left(\mu_{i}+\epsilon\right)\left\|t_{i}\right\|_{x, \epsilon}^{\prime}
$$

and

$$
\exp \left(\lambda_{i}-\epsilon\right)\left\|t_{i}\right\|_{g(x), \epsilon}^{\prime} \leq\left\|B_{i}\left(t_{i}\right)\right\|_{f^{n} \circ g(x), \epsilon}^{\prime} \leq \exp \left(\mu_{i}+\epsilon\right)\left\|t_{i}\right\|_{g(x), \epsilon}^{\prime} .
$$

We want to prove that $G_{1}$ has the same block diagonal form as $F_{1}$. Let us represent $G_{1}$ in the block form $G_{1}=\left(G_{i j}\right)$ and suppose $G_{i j} \neq 0$ for some $i \neq j$. Assume that $i>j$. It follows from (1.9) that

$$
G_{i j}\left(f^{n}(x)\right) \circ A_{j}^{n}=B_{i}^{n} \circ G_{i j}(x) .
$$

Let $h$ be a vector such that $G_{i j}(x) h \neq 0$, then for a large enough $n$ we have

$$
\begin{aligned}
& \left\|B_{i}^{n} \circ G_{i j}(x) h\right\|_{f^{n} \circ g(x), \epsilon}^{\prime} \geq \exp \left(n\left(\lambda_{i}-\epsilon\right)\right)\left\|G_{i j}(x) h\right\|_{g(x), \epsilon}^{\prime}> \\
& \exp \left(n\left(\mu_{j}+\epsilon\right)\right)\|h\|_{x, \epsilon}^{\prime}\left\|G_{i j}\right\| \geq\left\|G_{i j}\left(f^{n}(x)\right) \circ A_{j} h\right\|_{f^{n} \circ g(x), \epsilon}^{\prime}
\end{aligned}
$$

This contradicts (1.11). Similarly, If $i<j$ then $G_{i j}\left(f^{n}(x)\right) \circ A_{j}$ decreases slower than $B_{i} \circ G_{i j}(x)$. We proved that $G_{1}$ has the same form as $F_{1}$. Now we are going to apply induction. Assuming that $G_{n}$ has a sub-resonance generated form for all $n<k$, we'll prove that $G_{k}$ has also only sub-resonance generated terms.

Suppose it is not true. Let $J_{k}(G(x))=S(x)+N(x)$ where $S(x)$ is of subresonance type and $N(x)$ consists of non sub-resonance terms. By the assumption of induction we know that all terms of $N_{k}$ have degree $k$. We can also write down that

$$
\begin{gathered}
F^{n}(x)=A+\text { degree } \geq 2, \\
F^{n}(g(x))=B+\text { degree } \geq 2 .
\end{gathered}
$$

Let us rewrite (1.10) as

$$
\begin{gathered}
J_{k}\left(\left(S\left(f^{n}(x)\right)+N\left(f^{n}(x)\right)\right) \circ(A+\text { degree } \geq 2)\right)= \\
J_{k}((B+\text { degree } \geq 2) \circ(S(x)+N(x))) .
\end{gathered}
$$

Let us find terms which are not sub-resonance generated on the left and right hand sides of (1.12). We know that a composition of sub-resonance generated terms gives a sub-resonance generated term, so terms which are not subresonance generated and have degree $\leq k$ on the left hand side are of the form $N_{k}\left(f^{n}(x)\right) \circ A$ and from the right hand side of the form $B \circ N_{k}(x)$ So we have that

$$
N\left(f^{n}(x)\right) \circ A=B \circ N(x) .
$$


Let $N(x)=\left(N_{1}(x), \ldots, N_{l}(x)\right)$ where

$$
N_{i}(x): V(x) \rightarrow V_{i}(x) .
$$

It follows from (1.13) that

$$
N_{i}\left(f^{n}(x)\right) \circ A=B_{i} \circ N_{i}(x) .
$$

If $N_{i}(x) \neq 0$ then there exists a vector $t=\left(t_{1}, \ldots, t_{l}\right)$ such that $N_{i}(x) t \neq 0$ and the following inequality holds:

$$
\left\|B_{i} \circ N_{i}(x) t\right\|_{f^{n} \circ g(x), \epsilon}^{\prime} \geq \exp \left(n\left(\lambda_{i}-\epsilon\right)\right)\left\|N_{i}(x) t\right\|_{g(x), \epsilon}^{\prime}
$$

Now we estimate the norm of the vector $N_{i}\left(f^{n}(x)\right) \circ A t$. Each coordinate of this vector is a linear combination of products of $k$ coordinates of the vector At. It means each coordinate can be written as $\sum a_{s} \prod_{j}(A t)_{j}^{s_{j}}$ and all coefficients $a_{s}$ are bounded over $X$ (because they are derivatives at 0 ), also we know that $(A t)_{j}=\left(A_{i_{j}} t_{i_{j}}\right)_{j} \leq \exp \left(n\left(\mu_{i_{j}}+\epsilon\right)\right)\left\|t_{i_{j}}\right\|_{x, \epsilon}^{\prime}$. Each product can be estimated as $\exp \left(n \sum s_{j}\left(\mu_{i_{j}}+\epsilon\right)\right)\|t\|_{x, \epsilon}^{k}$. All products correspond to terms which are not sub-resonance generated so $\sum s_{j}\left(\mu_{i_{j}}+\epsilon\right) \leq \lambda_{i}-2 \epsilon$ and finally we have that

$$
\left\|N_{i}\left(f^{n}(x)\right) \circ A t\right\|_{g \circ f^{n-1}(x), \epsilon}^{\prime} \leq \operatorname{const} \exp (n(\lambda-2 \epsilon)) .
$$

where const doesn't depend on n. Comparing (1.16),(1.15) and (1.14) we get a contradiction, so $N(x)=0$ for all $x$ and induction is completed.

Step 2 . We proved in step 1 that $G(x)$ is a sub-resonance polynomial map, hence $G$ has a finite degree. We want to prove that $G=\tilde{\mathcal{G}}$ in some neighborhood of the zero section. Let us choose this neighborhood as a neighborhood of 0 such that $F=\tilde{\mathcal{F}}$ is a contraction and

$$
\|F t\| \leq \exp \left(\mu_{1}+2 \epsilon\right)\|t\|
$$

with respect to the standard $\epsilon$-Lyapunov metric (we will use only this metric up to the end of the proof so we omit the indices). The choice of this neighborhood depends only on first derivatives and can be done uniformly if they are continuous.

Suppose that for some $t$ from this neighborhood $\tilde{\mathcal{G}}(x) t \neq G(x) t$, so $\tilde{\mathcal{G}}(x) t=$ $G(x) t+h$. First we are going to prove that

$$
\Delta_{1}=\left\|F^{n}(g(x)) \circ \tilde{\mathcal{G}}(x) t-F^{n}(g(x)) \circ G(x) t\right\| \geq \operatorname{const} \exp \left(n\left(\lambda_{1}-\epsilon\right)\right)
$$

where const doesn't depend on $n$. Let $h=\left(h_{1}, \ldots, h_{l}\right)$ and $F^{n}(g(x))=$ $\left(C_{1}, \ldots, C_{l}\right)$. The linear part of $C_{i}$ is $B_{i}$ which was defined before. From the definition of sub-resonance terms it follows that $C_{1} h$ contains only linear terms 
and so $C_{1} t=B_{1} t_{1}$. So if $h_{1} \neq 0$ then $\Delta_{1}=\left\|B_{1} h_{1}\right\| \geq \exp \left(n\left(\lambda_{1}-\epsilon\right)\right)\left\|h_{1}\right\|$ and (1.14) is proved. If $h_{1}=0$ and $h_{2} \neq 0$ then we notice that all non-linear terms of $C_{2} t$ depend only on $t_{1}$, so $\Delta_{1}=\left\|B_{2} h_{2}\right\| \geq \exp \left(n\left(\lambda_{2}-\epsilon\right)\right)\left\|h_{2}\right\|$ and (1.18) is proved. We can continue these arguments and prove (1.18) for any $h \neq 0$.

On the other hand $\tilde{\mathcal{G}}$ and $G$ have the same derivatives at 0 , so for any $k>$ $\operatorname{deg} G$ and small enough $t$ we see that $\|\tilde{\mathcal{G}}(x) t-G(x) t\|=o\left(\|t\|^{k}\right)$. Moreover if $k$ - th derivatives are continuous then we can find a function $r(\|t\|)$ such that $\|\tilde{\mathcal{G}}(x) t-G(x) t\| \leq r(\|t\|)$ and $r=o\left(\|t\|^{k}\right)$.

We also see that (1.17) implies $\left\|F^{n}(x) t\right\| \leq \exp \left(n\left(\mu_{l}+2 \epsilon\right)\right)\|t\|$ so the following is true

$$
\begin{gathered}
\Delta_{2}=\left\|\tilde{\mathcal{G}}\left(f^{n}(x)\right) \circ F^{n}(x) t-G\left(f^{n}(x)\right) \circ F^{n}(x) t\right\|= \\
o\left(\left\|F^{n}(x) t\right\|^{k}\right) \leq \mathrm{const}\left\|F^{n}(x) t\right\|^{k} \leq \exp \left(n\left(k\left(\mu_{l}+2 \epsilon\right)\right)\right)\|t\|
\end{gathered}
$$

So if $k\left(\mu_{l}+2 \epsilon\right)<\lambda_{1}-2 \epsilon$ then the following is true

$$
\Delta_{2} \leq \text { const } \exp \left(n\left(\lambda_{1}-2 \epsilon\right)\right)
$$

Now $F^{n}$ commutes with $\tilde{\mathcal{G}}$ and $G$ so $\Delta_{1}=\Delta_{2}$, but it contradicts (1.18) and (1.19). So we see that $\tilde{\mathcal{G}}=G$.

\section{Diffeomorphisms with contracting foliations}

In what follows all "smooth" of " differentiable" objects are always assumed to be of class $C^{\infty}$ unless stated otherwise. A natural example of the situation described in Section 1 appears in hyperbolic dynamics. Let $M$ be a compact differentiable Riemannian manifold, $f: M \rightarrow M$ be a diffeomorphism of $M$. Let $\mathcal{W}$ be a continuous foliations with smooth leaves invariant under $f$. Let us also assume that $f$ contracts $\mathcal{W}$, i.e.

$$
\|D f \uparrow T \mathcal{W}\|<1
$$

We can consider $T \mathcal{W}$ as a vector bundle over $M$ and define the natural extension $\mathcal{N}_{f}$ of $f$ to a neighborhood of the zero section in $T \mathcal{W}$ by

$$
\mathcal{N}_{f} v=\exp _{f(x)}^{-1} \circ f \circ \exp _{x} v
$$

for $v$ in a ball of a fixed (sufficiently small) size about the origin in $T_{x} \mathcal{W}$. Let us denote these balls by $B_{x}$. Notice that we use the exponential map with respect to the induced Riemannian metrics on the leaves of the foliation $\mathcal{W}$, not for the global Riemannian metric on $M$. Under our assumption the natural extension is a contraction as an extension of $f$. Notice that although $f$ is smooth, since the foliation $\mathcal{W}$ was assumed to be only continuous, the natural extension is in general also only $C^{0}$. However, since the leaves of $\mathcal{W}$ are smooth the natural 
extension is smooth in the direction of the leaves. Now assume that $D\left(\mathcal{N}_{f}\right)_{0}$ (which is naturally identified with $D f \uparrow T \mathcal{W}$ ) has a narrow band spectrum (See Definition 1.1). Applying in this situation Theorem 1.2 we obtain a continuous family of smooth coordinate systems in the balls $B_{x}$, which can be projected by the exponentials to the balls on the leaves of $\mathcal{W}$. It turns out that on a fixed leaf those coordinate systems depend smoothly on the base point if all $V_{i}$ depend smoothly on the base point along a leaf. It can be seen from the proof of the Theorem 1.2. Since as we noticed above the natural extension of a smooth system is usually not differentiable there are no reasons to expect that $V_{i}$ change smoothly in the directions transversal to the leaves. However, even if extension itself is smooth the normal form in general does not depend smoothly on the base point.

Theorem 2.1. (Smooth dependence along leaves). Suppose that a manifold $M$ a diffeomorphism $f$ and a foliation $\mathcal{W}$ as above. Assume also that the natural extension $\mathcal{N}_{f}$ has a narrow band spectrum and all $V_{i}$ depend smoothly on the base point along a leaf. Then there exists a change of coordinates that conjugates $\mathcal{N}_{f}$ with an extension of sub-resonance type and is smooth along a leaf.

A complete proof can be found in $[\mathrm{G}]$. Here we only check that the formal change of coordinates found in step 1 depends on $x$ smoothly. As in the case of the existence of normal forms this is the crucial step. In order to do this we check that series (1.6) converge uniformly if we differentiate each element $\mathrm{k}$ times with respect to $x$. First we notice that the result consists of $\left(\begin{array}{c}2 n+1 \\ k\end{array}\right)$ sum of expressions of the form:

$$
M_{i}^{-1}(x) \ldots M_{i}^{-1}\left(f^{n}(x)\right) G_{f^{n}(x)}\left(M\left(f^{n-1}(x)\right) \ldots M(f(x))(M(x))(t)\right),
$$

where $M$ equals either $\Phi$ or its derivative and $G$ equals either $H$ or its derivative with respect to $x$. We also notice that at most $k$ factors will be different from what we had in (1.6) and so we can get

$$
\begin{gathered}
\left\|M_{i}^{-1}(x) \ldots M_{i}^{-1}\left(f^{n}(x)\right) G_{f^{n}(x)}\left(M\left(f^{n-1}(x)\right) \ldots M(f(x))(M(x))(t)\right)\right\| \leq \\
C\left\|\Phi_{i}^{-1}(x) \ldots \Phi_{i}^{-1}\left(f^{n}(x)\right) H_{f^{n}(x)}\left(\Phi\left(f^{n-1}(x)\right) \ldots \Phi(f(x))(\Phi(x))(t)\right)\right\|
\end{gathered}
$$

where $C$ depends only on $k$ and the maximum of the first $k$ derivatives along the leaves of the functions $\Phi \circ f^{i}$ and $H \circ f^{i}$ where $i=1, \ldots, n$. We know that $f$ is a contraction along the leaves so the maximum of the first $k$ derivatives for the function $f^{i}(x)$ goes to 0 when $i$ goes to infinity so maximum of the first $k$ derivatives for $\Phi\left(f^{i}(x)\right)$ and $H\left(f^{i}(x)\right)$ where $i=1, \ldots \infty$ is finite and so constant $C$ in the formula above does not depend on $n$. The convergence in (1.6) 
is exponential, so

$$
\begin{aligned}
\sum_{n=0}^{\infty} \| & M_{i}^{-1}(x) \ldots M_{i}^{-1}\left(f^{n}(x)\right) G_{f^{n}(x)}\left(M\left(f^{n-1}(x)\right) \ldots M(f(x))(M(x))(t)\right) \| \\
& \leq \sum_{n=0}^{\infty} C \exp (-\alpha n)
\end{aligned}
$$

and therefore all the derivatives converge uniformly.

\section{Applications to the rigidity of group actions}

The first principal application of the version of the theory of non-stationary normal forms developed in this paper is in the proof of local differentiable rigidity of algebraic Anosov actions of higher-rank abelian groups. [KS, Theorem 1, see Section 2.2.3 Step 4]. The crucial point is to prove that a Hoelder conjugacy between the orbit foliations of the algebraic system and its perturbation is smooth along the leaves of certain invariant foliations. The normal form and centralizer theorems provide invariant families of "geometric structures" on the leaves of those foliations for both the original algebraic system and its small perturbation. This allows one to conclude that the conjugacy intertwines a transitive family of diffeomorphisms on the fibers and eventually to deduce smoothness of the conjugacy. Rigidity of the abelian actions is further used in [KS Sections 3 and 4] to establish local differential rigidity of two very different types of algebraic actions of cocompact lattices in higher rank semisimple Lie groups: Anosov actions by automorphisms of tori and (infra)nil-manifolds and projective actions on the homogeneous spaces of parabolic subgroups. The same scheme is followed in another very recent significant application of our non-stationary normal forms theory, namely the proof of local differentiable rigidity of algebraic weakly hyperbolic actions of higher rank semisimple Lie groups and lattices in such groups by G.A Margulis and N. Qian ([MQ, Section 5]) which in particular extends the rigidity of actions on tori and (infra)nil-manifolds beyond the Anosov case.

A different version of the non-stationary normal forms theory is outlined by the second author in [K2]. It deals with the measurable non-uniformly hyperbolic situation, i.e. with a diffeomorphism preserving a measure with nonvanishing Lyanunov characteristic exponents (a hyperbolic measure). In a sense the situation is simpler because the multiplicative ergodic theorem allows to consider literal resonances between the exponents. Nevertheless the notion of a sub-resonance relation remains crucial. The counterparts of the normal form and centralizer theorems from [K2] are used to establish rigidity properties for hyperbolic measures for smooth actions of higher rank abelian groups. Under certain conditions positivity of entropy for certain elements of the action in question implies that the measure must be absolutely continuous. This allows among 
other things to deduce that any smooth $\mathbb{Z}^{k-1}$ action on the torus $T^{k}, k \geq 3$ homotopic to an action by hyperbolic automorphisms (i.e. hyperbolic on the first homology group) preserves an absolutely continuous measure.

Some of the results of this paper have been announced in [KS2].

\section{References}

[BK] I. U. Bronstein and A. Ya. Kopanskii, Smooth invariant manifolds and normal forms, World Scientific, 1994.

[C] K.-T. Chen, Equivalence and decomposition of vector fields about an elementary critical point, Amer. J. of Math. 85 (1963), 639-722.

[DL1] D. DeLatte, Nonstationary normal forms and cocycle invariants, Random and Comp. Dyn. 1 (1992-93), 229-259.

[DL2] - On normal forms in Hamiltonian dynamics, a new approach to some convergence questions, Erg. Theory and Dynam. Syst. 15 (1995), 49-66.

[F] R. Feres, The invariant connection of a 1/2-pinched Anosov diffeomorphism and rigidity, Pacific J. Math (1995).

[G] M. Guysinsky, The theory of non-stationary normal forms, in preparation.

[K1] A. Katok, Local properties of hyperbolic sets (in Russian), Supplement to the Russian translation of "Differentiable Dynamics" by Z.Nitecki, Mir, Moscow, 1975, pp. 214-232.

[K2] - Hyperbolic measures for actions of higher-rank abelian groups, preprint (1996).

$[\mathrm{KH}]$ A. Katok and B. Hasselblatt, Introduction to the modern theory of dynamical systems, Cambridge University Press, 1995.

[KS1] A. Katok and R. Spatzier, Differential rigidity of Anosov actions of higher rank abelian groups and algebraic lattice actions, Proc. Steklov Math. Inst. 216 (1997), 287-314.

[KS2] , Non-stationary normal forms and rigidity of group actions, Electr. Res. Ann. of the AMS 2 (1996), 124-133.

[MQ] G. A. Margulis and N. Qian, Local rigidity of weakly hyperbolic actions of higher rank semisimple Lie groups and their lattices, preprint (1997).

[Q] N. Qian, The existence and uniqueness of measurable invariant invariant affine connection under Anosov diffeomorphism, preprint (1992).

[S] S. Sternberg, Local contractions and a theorem of Poincaré, Amer. J. of Math. 79 (1957), 809-824.

Dept. of Math., Pennsylvania State University, University Park, PA 16802 USA

E-mail address: guysin_m@math.psu.edu, katok_a@math.psu.edu 